\title{
Development of projects reference framework for a French-Arabic- French translation program
}

\section{[Elaborer un referentiel des projets pour une formation de traduction francais-arabe-francais]}

\author{
Rana Kandeel
}

DOI: 10.18355/XL.2019.12.01XL.15

\begin{abstract}
This article presents the results of an analysis of the components of context of development of French language projects reference framework in a translation program studies for students of French as a foreign language (FFL). The study falls into two fields: the didactics of FFL and the didactics of translation. It aims to identify and analyze the essential components of the reference framework (contents, skills, tasks and evaluation criteria's), through the analysis of linguistic and professional reference frameworks and through the discourse of students in a university context about the implemented projects and those that will be achieved in translation program studies. The research methodology is quantitative and qualitative to understand the students' discourse about the utilization the projects. The results of the study lead to the identification of the components of the reference framework and to the production of a document model of the project reference framework.
\end{abstract}

Key words: contents, skills, project, program of translation studies, FFL learners, reference framework

\section{Résumé}

Cet article présente les résultats d'une analyse des composantes du contexte d'élaboration du référentiel des projets en français pour une formation universitaire de traduction destinée à des étudiantes en français langue étrangère (FFL). L'étude s'inscrit dans deux champs disciplinaires : la didactique du FLE et la didactique de la traduction. Elle vise à identifier et à analyser les composantes essentielles du référentiel (contenus, compétences, tâches et critères d'évaluation), à partir de l'analyse des cadres des références linguistiques et professionnelles et du discours des étudiantes sur les projets en français mis en œuvre et à réaliser dans la formation de traduction. La méthodologie de la recherche est quantitative et qualitative pour comprendre le discours des étudiantes sur l'utilisation des projets. Les résultats de l'étude ont conduit à l'identification des composantes du référentiel et à la production d'un modèle de document du référentiel des projets.

Mots-clés : contenus, compétences, projet, formation de traduction, apprenants FLE, référentiel

\section{Introduction}

L'élaboration des référentiels des formations en langues étrangères est une pratique institutionnelle qui nécessite un renouvellement fréquent des maquettes des formations, en raison de l'évolution des besoins des apprenants, des pratiques des enseignantes et d'une organisation de l'activité formative. Elle entraîne des ajustements sur l'apprentissage des langues par la construction d'un ensemble de repères partagés pour la mise en œuvre des savoirs et des compétences acquis. Les savoirs sont les connaissances qui résultent de l'expérience (savoirs empiriques) ou d'un apprentissage plus formel (savoirs académiques). Les compétences désignent 
"l'ensemble des connaissances, des habiletés et des dispositions qui permettent d'agir" (CECERL, 2001 : 15).

Dans le cadre d'une formation à la traduction, dans un contexte FLE, il s'agit de former des acteurs sociaux capables de réaliser des projets en français à visée professionnelle, afin de les préparer à travailler dans des milieux professionnels de plus en plus internationalisés. C'est pourquoi la nécessité de concevoir les projets et de les définir dans un cadre de référence précis devient incontournable pour renforcer les liens entre les formations et les compétences à mobiliser dans une situation professionnelle donnée.

Les études sur les référentiels en formation ont fait l'objet de différents travaux. Au cœur du questionnement se trouvent les usages qu'en font les acteurs, les formateurs et les praticiens (Leclerq - Vicher, 2012), les degrés de convergence ou de divergence des référentiels avec les activités visées et leurs usages effectifs (Cadet, 2014). Dans cet article, nous souhaitons soulever la question de l'élaboration du référentiel des projets destiné aux formations de traduction pour un public d'apprenants du FLE. Nous nous intéressons, tout particulièrement à l'identification de ses composantes essentielles, à définir d'après l'analyse des cadres de référence des langues et des compétences.

Notre article s'articule autour de quatre axes. Le premier est consacré à la définition du référentiel en didactique des langues et à son élaboration dans une formation de traduction. Après la présentation du contexte de la recherche et de la problématique, nous abordons la partie de la méthodologie de la recherche, qui comprend le corpus, les données collectées ainsi que l'analyse des données. Le troisième axe concerne les résultats de l'analyse et la discussion. Enfin, le dernier présente la production d'un modèle de document du référentiel des projets.

\section{Le référentiel en didactique des langues}

Le référentiel est défini comme "un inventaire de compétences nécessaires à des activités et l'inventaire finalisé de ces activités elles-mêmes" (Cuq, 2003 : 212). Cette définition met l'accent sur la notion de compétences, en la reliant aux activités langagières de l'apprenant. Les exemples des référentiels ne manquent pas dans le domaine des langues et dans la formation du FLE/FOS ${ }^{i}$. Ils sont destinés aux professeurs de langues pour synthétiser les savoir-faire, les contenus lexicaux, grammaticaux et communicatifs à enseigner, les aspects socioculturels à traiter et les modalités d'évaluation ou de certification.

Quand la formation en langue a pour objectif de préparer les apprenants à un domaine particulier de travail tel que la traduction, le référentiel devient un document indispensable auquel un enseignant se réfère pour établir une relation entre le champ de formation et le champ de pratique professionnelle (Rey, 2010 :118). Il permet à l'apprenant de mettre en œuvre des connaissances et des compétences acquises durant la formation dans ses futures activités professionnelles. Or, le processus d'acquisition d'une compétence est circulaire (CECRL, 2018 : 33), c'est-à-dire que l'apprenant développe ses compétences et acquiert des stratégies en réalisant des activités au cours de sa formation. Comme le référentiel est conçu pour guider l'action de l'enseignant, il s'agit donc de l'utiliser pour aider les apprenants à devenir des acteurs sociaux dans l'usage et l'apprentissage de la langue, tel que le préconise le CECRL. 
Le CECRL met l'accent sur le mot-clé domaine car il comprend des catégorisations relatives à l'enseignement/apprentissage des langues dans la formation académique à visée professionnelle. Il met en évidence la relation entre les savoirs, les compétences, les activités et les contextes de leur pratique. Si le contexte de pratique est professionnel et qu'il fait face à la concurrence, les spécialistes de la formation procèdent alors à l'élaboration des référentiels conçus pour augmenter la qualité de la formation.

Les référentiels ne sont pas tous identiques car ils varient en fonction de leurs méthodes de réalisation, de leur terminologie, de leurs cadres théoriques de références, de leurs fonctions (Leclercq - Vicher, 2012 :96) et du public auquel ils s'adressent. Qu'il soit un référentiel d'activités, de formation, ou de compétences, un référentiel est élaboré dans le but d'avoir un impact sur le contenu et les modalités de mise en œuvre de l'activité formative (Chauvigné, 2010 :77). Selon Leclercq et Vicher : "Ces supports ont une fonction de cadrage global, fournissent des repères pour la définition de politiques de formation, donnent une direction dans laquelle doit se dérouler l'intervention éducative et précisent davantage les finalités des formations que les objectifs précis" (2012:79).

\section{Elaboration d'un référentiel des projets dans la formation de traduction}

Un référentiel des projets est un document support qui répertorie les projets à réaliser dans une formation de traduction en formalisant les compétences langagières et professionnelles attendues à la fin des modules de formation, à travers la description des tâches indispensables à la réalisation des objectifs de la formation. Il situe les compétences selon des cadres de références de langues, de compétences et de professions dans un contexte donné et selon les niveaux des apprenants dans l'échelle des descripteurs du CECRL, en y mentionnant les critères d'évaluation.

\section{1. Pourquoi élaborer un référentiel des projets ?}

L'élaboration d'un référentiel des projets entre dans le processus de professionnalisation des enseignants de langue tant pour les novices que pour les plus expérimentés. Il constitue aussi un guide pour les apprenants dans leur formation, ainsi qu'un indicateur de qualité de la formation au niveau institutionnel puisqu'il guide l'activité de formation et sert à son évaluation.

Le référentiel des projets permet de formaliser les projets et de les orienter vers l'action en complétant le rôle du référentiel de formation. Ce dernier est défini comme "le document qui indique aux enseignants les objectifs, les contenus sur lesquels ils doivent faire travailler les apprenants, et qui donne un certain nombre d'instructions pédagogiques pour sa mise en œuvre" (Cros - Raisky, 2010 : 109). Le référentiel joue un rôle de réconciliateur entre les projets et les programmes de formation (Christin, 1997 : 27). C'est donc un outil facilitateur du travail des enseignantes et des coordinatrices pédagogiques quand elles ne sont pas formées à la pédagogie du projet. D'ailleurs, certaines enseignantes n'ont pas pratiqué pendant leur formation initiale une expérience de conception d'un projet, qu'il soit pédagogique ou professionnel. En introduisant des balises plus précises, le référentiel constitue un langage commun pour traiter de l'acte professionnel (Lenoir, 2010 : 92), ce qui réduit les attitudes d'incertitude auxquels les enseignantes sont confrontées.

Considéré comme une ressource indispensable à la conception et à la mise en place des actions (Leclercq - Vicher, 2012 : 94), cet outil de rationalisation et de cadrage,

XLinguae, Volume 12 Issue 1XL, January 2019, ISSN 1337-8384, eISSN 2453-711X 
souvent élaboré selon des normes et critères internationaux, contribue à l'homogénéisation des types de projets à réaliser selon les spécialités des formations. Il permet une cohérence entre les connaissances enseignées et les objectifs escomptés de l'apprentissage. Dans la formation à la traduction (français-arabe-français) à l'Université Princesse Nourah Bint Abdulrahman en Arabie Saoudite, où le français est enseigné comme une langue étrangère au cours des deux premières années, puis, comme une langue de spécialité (traduction) au cours des deux dernières, l'élaboration du référentiel des projets est indispensable pour mettre en place un nouveau type de tâches dans la formation des étudiantes FLE. Les tâches mises en œuvre dans les deux premières années de formation sont réalisées en français et non pas dans les deux langues, le français et l'arabe. Par exemple, la rédaction de textes simples est réalisée en français, sans vérifier que l'étudiante soit capable de le traduire en arabe. Par conséquent, les étudiantes se retrouvent confrontées à la traduction de textes longs à partir de la troisième année, sans une préparation préalable dans les deux premières années à l'activité de traduction.

Il s'agit donc d'introduire de nouvelles tâches centrées sur les activités de médiation des textes. Les activités sont présentées par le CECRL (2001 : 18) et son volume complémentaire publié en 2018 par le conseil de l'Europe. Les quatre types de médiation présents dans le CECRL sont linguistiques, culturelles, sociales et pédagogiques (North - Piccardo, 2016 : 13). Selon le CECRL, les activités de médiation "permettent, par la traduction ou l'interprétariat, le résumé ou le compte rendu, de produire à l'intention d'un tiers une (re)formulation accessible d'un texte premier auquel ce tiers n'a pas d'abord accès direct. Les activités langagières de médiation, (re)traitant un texte déjà là, tiennent une place considérable dans le fonctionnement langagier ordinaire de nos sociétés" (2001 : 18). Les tâches sont à harmoniser avec les connaissances des descriptifs de la formation en question. Or, le moment privilégié de la mise en œuvre des compétences acquises pendant la formation est la réalisation des tâches du projet.

\subsection{Les étapes de l'élaboration du référentiel des projets}

Pour élaborer un référentiel de projet, nous suivons plusieurs étapes :

- Identifier les modules de la maquette de formation qui intègrent le projet.

- Analyser les descriptifs : place et fonction des projets.

- Interroger les étudiantes sur les contenus des projets mis en œuvre.

- Etablir un lien entre les contenus des projets et les cadres de références afin de vérifier leur adéquation avec les contenus et les compétences du volume complémentaire du Cadre Européen Commun de Référence pour les langues (CECRL), le référentiel de compétences de l'EMT, et le guide local de catégorisation des professions en Arabie Saoudite.

- Interroger les étudiantes sur leurs attentes vis-à-vis des types des projets à réaliser dans le cadre de la formation de traduction.

- Identifier les composantes du référentiel en fonction des résultats de l'analyse.

\section{Contexte de la recherche}

Le référentiel est à élaborer pour une formation de traduction dans le programme de la langue française du département de traduction à l'Université Princesse Nourah bint Abdulrahman en Arabie Saoudite. Les enseignantes sont de rang magistral, francophones et de spécialités différentes : traduction, linguistique, sciences du langage, littérature et didactique et de diverses nationalités ; française, algérienne, tunisienne, égyptienne et jordanienne. Elles ont toutes un doctorat dans des spécialités 
variées : traduction, linguistique, sciences du langage, littérature et didactique. La variété de leurs spécialités donne une richesse linguistique et culturelle à la formation. Certaines ont une expérience d'enseignement qui dépassent vingt ans et d'autres sont au début de leur carrière professionnelle.

Quant aux étudiantes, la majorité d'entre elles commencent leurs études en français à l'Université sans aucune connaissance de cette langue, c'est la raison pour laquelle la grande majorité d'entre elles ont le niveau linguistique A2 à la fin de leurs études. La langue française est la deuxième langue étrangère en Arabie Saoudite. La première étant l'anglais, et l'arabe est la langue maternelle des étudiantes. Elles apprennent le français pendant quatre ans pour décrocher une licence de traduction ${ }^{\text {ii }}$. Les deux premières années représentent quatre niveaux d'enseignement et chaque niveau est réparti sur un semestre d'études de quinze semaines. Les modules de ces niveaux visent les connaissances linguistiques. Les deux dernières années visent à la fois les connaissances et les compétences disciplinaires et professionnelles (tableau 1). Dans le cadre de cette recherche, nous allons analyser les projets dans les descriptifs des modules des cinq premiers niveaux. 


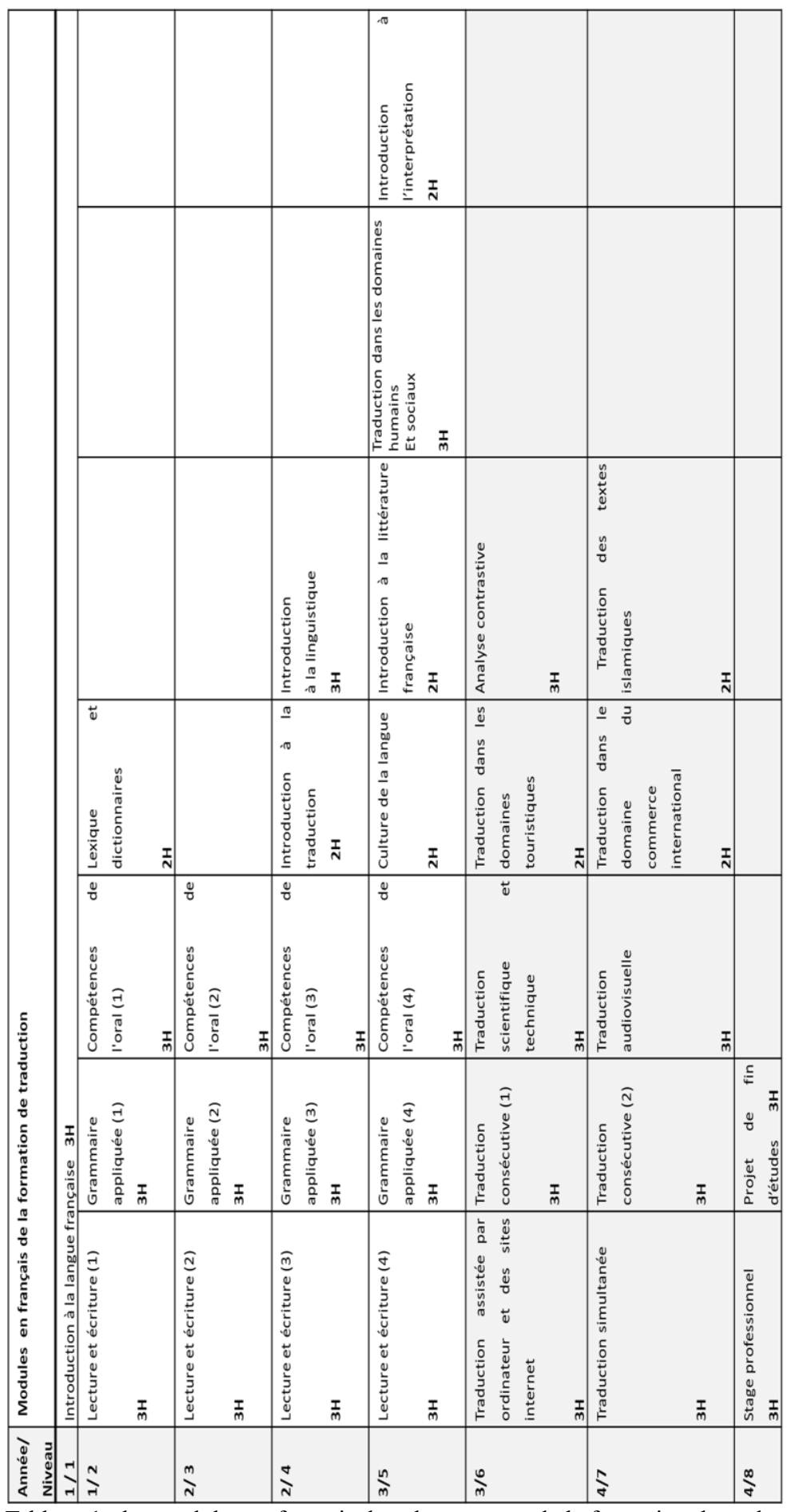

Tableau 1 : les modules en français dans la maquette de la formation de traduction 


\section{Problématique de la recherche}

L'intérêt de la pédagogie du projet est incontournable et approuvé par les spécialistes de la traduction (Martinez Melis, 2001 ; Hutrado Albir, 2008) et des langues étrangères (Bougherra, 1996 ; Christin, 1997 ; Bracke, 2001 ; Puren, 2009, Hamez, 2012). Pour les premiers, le projet joue un rôle primordial dans une formation à la traduction. Il permet de mettre en œuvre les connaissances et les compétences de traduction. La compétence de traduction est une connaissance procédurale qui ne s'acquiert que par la pratique, et par son utilisation d'une manière efficace dans un contexte donné. Cette compétence fait partie de l'ensemble des compétences professionnelles nécessaires à la pratique du métier de traducteur. Une compétence professionnelle désigne "un savoir-faire complexe (connaissances, habiletés, attitudes, valeurs, etc.) qui garantit l'efficacité d'un exercice professionnel" (Hutrado Albir, 2008: 20).

Pour les seconds, le projet constitue un levier de l'apprentissage pour acquérir une langue étrangère dans une dimension sociale. C'est un intégrateur didactique, un outil privilégié pour la mise en place d'une approche didactique fondée sur les principes de l'approche actionnelle. Les principes de cette approche placent l'apprenant au centre de l'apprentissage et l'incitent à réaliser des actions sociales où la langue étrangère devient le moyen de communication.

Un référentiel des projets est identique au référentiel de compétences dans sa logique de conception, dans la mesure où il exige que l'institution conçoive le référentiel selon une logique de l'action et d'une professionnalisation du métier (Lenoir, 2010 : 93). Mais il diffère du référentiel de compétence par la définition de la thématique du projet. Pourtant, le choix des projets en fonction de leurs contenus et compétences visées, dans une formation de traduction où les apprenants ont des niveaux linguistiques limités, ne semble pas une action aisée pour les enseignants et les apprenants.

Notre observation de terrain, en tant qu'enseignante de FLE dans une formation de traduction à l'Université Princesse Nourah bint Abdulrahman, nous a permis de constater une incohérence, d'une part, entre certains projets mis en œuvre et les objectifs des modules, ce qui conduit à des choix peu adéquats des types de projets à réaliser, et d'autre part, entre les types de projets proposés par les enseignantes des modules à visée linguistique et les objectifs de la formation dans le domaine professionnel de la traduction. En effet, le projet est évoqué dans le descriptif sans pour autant être défini.

Ce constat nous a conduit à élaborer un référentiel des projets pour les définir en adéquation avec les objectifs de la formation de traduction français-arabe, les attentes des apprenants ainsi qu'avec les ouvrages de références internationaux spécialisés en langues étrangères et en traduction. Mais l'élaboration d'un référentiel devrait passer par des étapes structurées reposant sur des analyses des éléments qui le composent.

Notre problématique de recherche porte donc sur l'analyse et l'identification des composantes du référentiel des projets. Il ne s'agit pas d'élaborer le référentiel dans le cadre de cette recherche, mais de produire un document modèle du référentiel des projets afin de le tester dans les cours. Pour aborder notre problématique, nous sommes partie des questions suivantes :

1. Quelles sont les composantes à analyser pour établir les matériaux du référentiel?

XLinguae, Volume 12 Issue 1XL, January 2019, ISSN 1337-8384, eISSN 2453-711X 
2. Quels sont les cadres de références pour cette élaboration?

3. Quels sont les projets mis en œuvre sur le terrain dans les cinq premiers niveaux de formation? Quels sont leur type (linguistique ou traduction) et leur contenus?

4. Quel est le degré de cohérence des contenus des projets mis en œuvre avec les objectifs de la formation? Avec les compétences visées par le Référentiel de compétences de l'EMT? Avec le CECRL et son volume complémentaire ? Avec le guide de catégorisation des professions en Arabie Saoudite?

5. Quels sont les attentes des apprenants vis-à-vis des types de projets à réaliser dans la formation de traduction?

6. Quels sont les éléments à intégrer dans le référentiel ?

Afin de répondre aux questions de recherche, nous avons adopté une méthodologie combinée quantitative-qualitative reposant sur la collecte des données et des sources indispensables à la réalisation du référentiel.

\section{Méthodologie de la recherche}

\subsection{Collecte des données :}

Les données collectées sont de deux types : corpus et cadre de référence.

\subsubsection{Le corpus}

Le corpus comprend les descriptifs des programmes de langue française pour les cinq premiers niveaux et les informations recueillies sur les projets dans le cadre d'une enquête du terrain.

Les descriptifs sont élaborés par l'équipe d'enseignantes francophones à l'Université, ils ont pour objet de décrire les savoirs et les compétences à faire acquérir aux étudiantes, les stratégies d'enseignement, les résultats escomptés de l'apprentissage ainsi que les modalités d'évaluation. Vingt descriptifs ont été analysés pour recueillir uniquement ceux qui intègrent la notion de projet dans leur conception, car le but ultime de l'élaboration du référentiel est de répondre à un besoin de définition des projets afin de les proposer selon les critères des cadres internationaux. Notre choix de ces cinq niveaux est motivé par la volonté de développer les activités d'apprentissage des projets de la nouvelle maquette de formation qui a succédé à l'ancienne maquette depuis environ trois ans. Cette nouvelle formation est à mi-parcours. Les projets sont principalement proposés dans ces modules.

Les informations sont recueillies sur les projets dans le cadre d'une enquête réalisée auprès de soixante-sept futures traductrices inscrites au cinquième niveau à l'Université Princesse Nourah bint Abdulrahman. Elles permettent de définir les contenus des projets mis en œuvre, et d'identifier les attentes à prendre en compte dans le processus de référentialisation, tout particulièrement les types de projets à mettre en place dans la formation selon le discours épilinguistique des étudiantes.

L'enquête a été menée au moyen d'un questionnaire ayant pour objectif l'identification des projets mis en œuvre. Ce questionnaire a été envoyé aux étudiantes de cinquième niveau par mail en septembre 2018. Le questionnaire a été élaboré en arabe, la langue maternelle des étudiantes, afin de faciliter la compréhension des 
questions et de leur permettre de bien dire ce qu'ils pensent. Il comprend deux grandes parties : la première est consacrée au profil des futures traductrices, à l'identification des projets, aux attentes des étudiantes vis-à-vis des types de projets à réaliser. La deuxième partie concerne les contenus des projets mis en œuvre. Nous avons eu le retour de quarante-sept questionnaires.

\subsubsection{Cadres de référence}

Un référentiel présente les buts à atteindre et les normes à respecter dans la réalisation des projets pour guider l'action pédagogique des enseignants. Pour bien formuler le référentiel, le point de départ de l'élaboration doit être la formulation des principales caractéristiques qui définissent les performances dans ce domaine (North \& Piccardo, 2016 : 36). C'est la raison pour laquelle nous avons eu recours aux cadres de référence afin de définir les contenus et les compétences des projets, à savoir : le volume complémentaire du CECRL (2018), le Référentiel de compétences de l'EMT, le guide de catégorisation des professions en Arabie saoudite (figure 1). Les descriptifs de formation et les résultats de l'enquête constituent les autres sources de référence indispensable à l'élaboration.

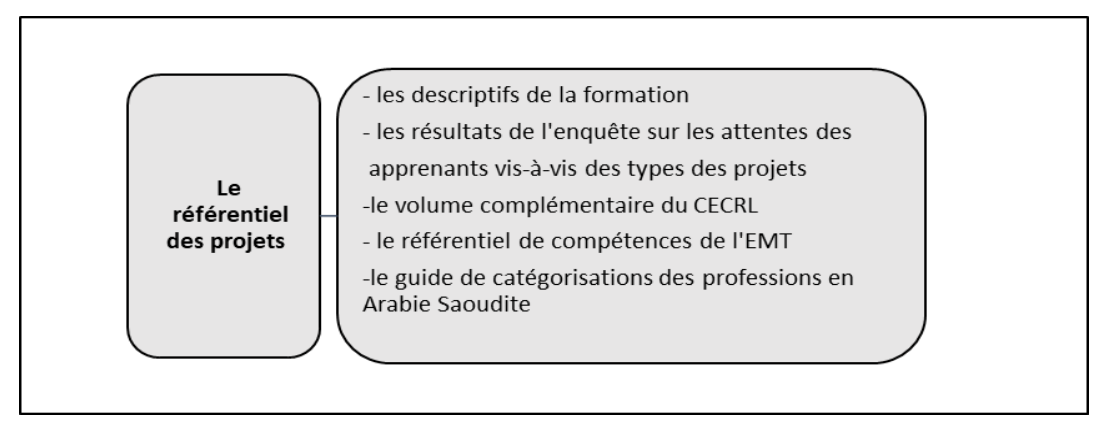

Figure 1 : cadre de référence pour l'élaboration du référentiel

Le CECRL dresse les niveaux de compétences permettant de mesurer les progrès de l'apprenant à chaque étape de l'apprentissage de langue (CECRL, 2001 : 9). Il est utilisé comme indicateur du niveau de compétence langagière. Il a été complété par un descripteur des activités de médiation où la traduction à l'oral et à l'écrit des textes écrits est considérée comme une activité de médiation de textes. Les notions clés concrétisées dans l'échelle pour la traduction à l'oral d'un texte écrit pour les niveaux inférieurs sont :

- $\quad$ assurer une traduction sommaire, approximative.

- $\quad$ saisir l'information essentielle (CECRL, 2018 :117).

De manière similaire, les notions-clés concrétisées pour ces niveaux pour la traduction d'un texte à l'écrit comprennent :

- intelligibilité de la traduction ;

- mesure dans laquelle les formulations et structures du texte d'origine (sur-) influencent la traduction, au contraire du texte qui respecte les conventions pertinentes dans la langue cible (CECRL, 2018: 117). 
Le choix de ce cadre de référence permet de légitimer le choix des activités de traduction à proposer dans les projets. Selon les nouveaux descripteurs du CECRL, les deux activités destinées à notre public ayant les niveaux A1 et $\mathrm{A} 2$ sont les suivantes :

Traduire à l'oral un texte écrit est une activité complètement informelle qui n'a rien d'inhabituel dans la vie personnelle et professionnelle de tout un chacun. Il s'agit de traduire oralement, de façon spontanée, un texte écrit, souvent un message, une lettre, un courriel ou toute autre communication...Traduire à l'écrit un texte écrit relève, de par sa nature même, d'un processus beaucoup plus formel que traduire à l'oral (CECRL, 2018 :117).

Il est important de signaler que l'échelle est centrée sur les compétences langagières. Pour combler le manque de traitement de la compétence en traduction, nous avons eu recours au référentiel de compétences de l'EMT (Commission européenne, 2017) qui définit la compétence traductionnelle comme la capacité des apprenants à découvrir divers aspects relatifs à la prestation de services de traduction, à l'extraction de l'information, ainsi qu'à l'acquisition des compétences linguistiques, interculturelles, technologiques et thématiques. Ces compétences de base doivent être acquises par le traducteur. Les universités de l'Union Européenne et au-delà de cette zone, se servent de ce cadre comme modèle pour élaborer leurs programmes.

En dernier lieu, la consultation du guide de catégorisation des professions en Arabie Saoudite nous a permis d'identifier les noms des catégories de profession à exercer dans le domaine de la traduction. Ce guide donne une brève présentation de l'ensemble des catégories, des savoirs et des compétences exigées, et le niveau de diplôme nécessaire à l'exercice de l'emploi ${ }^{\text {iii }}$.

\section{L'analyse des données}

L'analyse des données est quantitative et qualitative. L'analyse quantitative est réalisée selon trois axes : tout d'abord, les profils des futures traductrices qui ont participé à l'enquête : âge et nombre d'années d'apprentissage du français, l'utilisation des projets dans leur apprentissage, ensuite, le taux d'utilisation, instructions données sur les projets et connaissance des critères d'évaluation et enfin le troisième axe se concentre sur les attentes des étudiantes vis-à-vis des types de projets à réaliser dans la formation.

Nous avons réalisé l'analyse qualitative de cinq dimensions : les descriptifs, les réponses à la question du questionnaire sur les contenus des projets mis en œuvre, le cadre des compétences du référentiel, celui des compétences de l'EMT, le volume complémentaire du CECRL et enfin le guide de catégorisation des professions en Arabie Saoudite. Chaque catégorie est analysée selon les axes thématiques suivants :

$\rightarrow$ Les descriptifs : types de module intégrant le projet, place du projet, informations sur son contenu/sujet, informations sur les modalités de sa mise en œuvre, et de ses modalités d'évaluation.

$\rightarrow$ Réponses au questionnaire : contenus des projets mis en œuvre, attentes des étudiantes vis-à-vis des projets à réaliser.

$\rightarrow$ Référentiel de compétences de l'EMT : compétences nécessaires au traducteur pour entrer dans le marché de travail. 
$\rightarrow$ CECRL et son volume complémentaire : compétences langagières et activités de médiation.

$\rightarrow$ Guide de catégorisation des professions en Arabie Saoudite : contenus et compétences.

C'est l'ensemble de ces données que nous avons utilisées afin d'identifier les composantes du référentiel avant de commencer son élaboration.

\section{Résultats de l'analyse}

\subsection{Analyse quantitative : enquête par questionnaire}

\subsubsection{Profils des étudiantes}

La majorité des étudiantes qui ont participé à l'enquête font partie de la tranche d'âge des 21-23 ans (tableau 2), c'est-à-dire qu'elles ne sont pas débutantes en langue lors de la réalisation de l'enquête. Elles ont une expérience d'apprentissage leur permettant de proposer, après deux ans d'études de la langue étrangère, les projets reliés à la discipline de la traduction et dont elles ont besoin pour développer leur apprentissage. Le nombre d'années d'apprentissage du français est de deux ans pour la majorité des étudiantes interrogées (tableau 3 ). Ce résultat montre que les projets à proposer, dont certains vont figurer dans le référentiel, doivent tenir compte aussi de l'histoire de l'apprentissage du français par les étudiantes. Pour elles, le français est appris pour la première fois à l'Université, et même si les étudiantes suivent une formation de traduction, les contenus doit convenir à leur niveau.

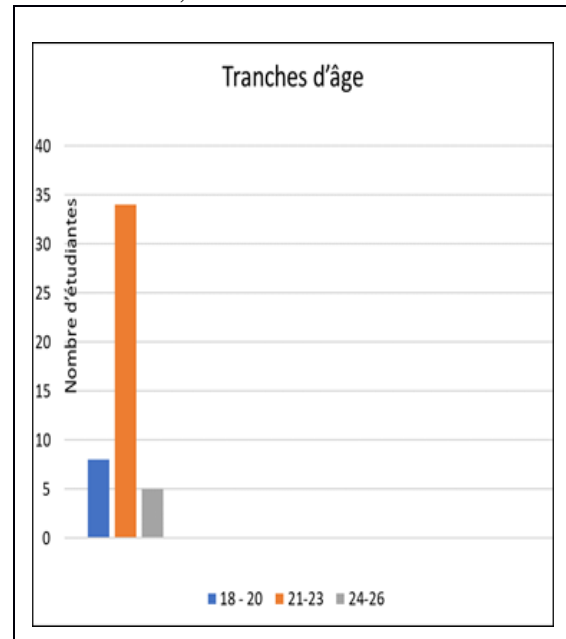

Tableau 2 : âges des étudiantes

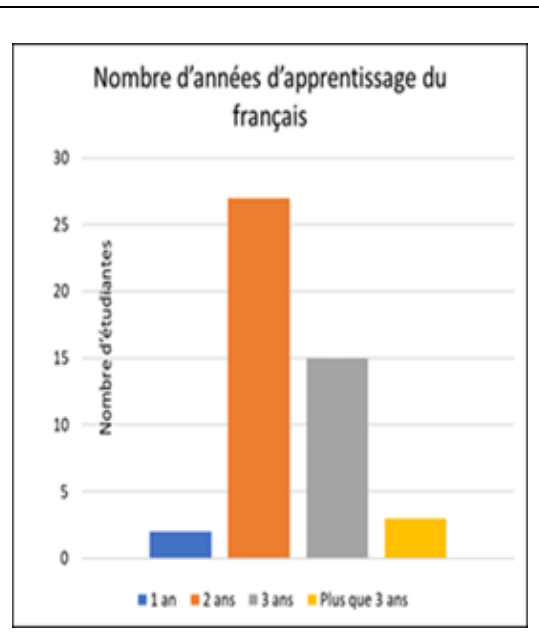

Tableau 3 : années d'apprentissage

\subsubsection{Utilisation des projets dans la formation à la traduction}

La plupart des étudiantes ont répondu avoir utilisé les projets dans les cours de français. C'est-à-dire que les projets sont mis en œuvre dans le cadre du programme 
(tableau 4), et peu d'entre elles ont répondu ne pas avoir utilisé les projets, cela peut être expliqué par le fait que dans notre échantillon, certaines étudiantes ont été inscrites dans l'ancien programme d'étude, qui n'a pas proposé d'implémenter ce type d'activité en classe de langue.

Concernant la question sur les instructions données par l'enseignante concernant la réalisation du projet, la quasi-totalité des étudiantes disent qu'elles les ont reçues (tableau 5). Les enseignantes expliquent ces instructions en classe ou elles les leur envoient par mail.

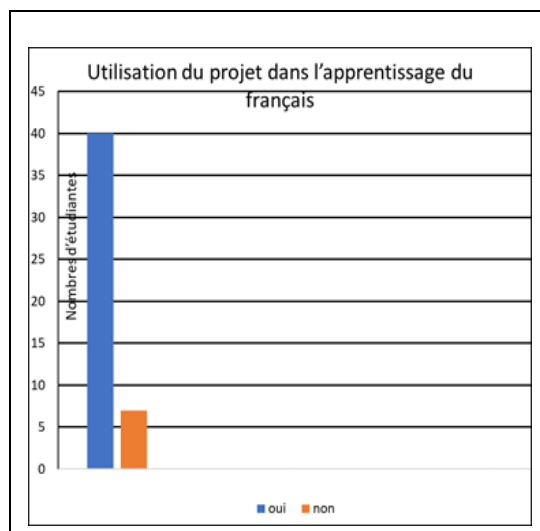

Tableau 4 : projet et apprentissage

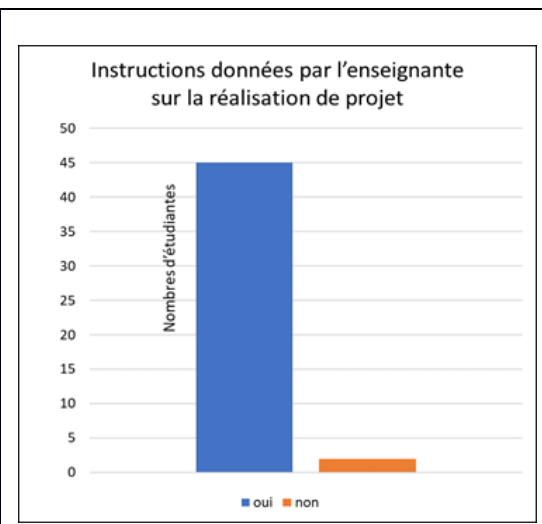

Tableau 5 : instructions et réalisation du projet

Les étudiantes confirment leur connaissance des critères d'évaluation des projets (tableau 6). Bien que la note accordée au projet soit évoquée dans les descriptifs, mais aucune autre référence ne donne des informations sur les critères d'évaluation. Les enseignantes proposent ces critères sans qu'il y ait une référence de l'institution sur ces critères.

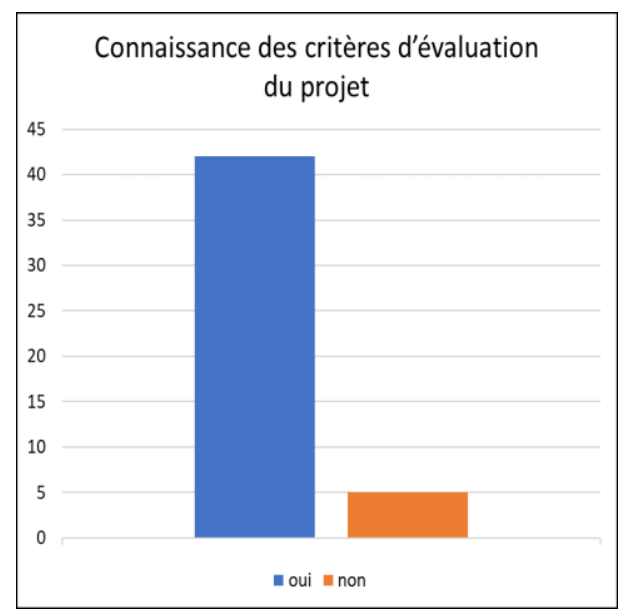

Tableau 6 : critères d'évaluation 


\subsubsection{Les attentes des étudiantes vis-à-vis des types de projets à réaliser dans la formation de traduction}

Les réponses des étudiantes à la question sur les types de projets à réaliser pour répondre à leurs attentes dans la formation à la traduction ont montré que vingt-six étudiantes veulent travailler sur des projets de traduction (figure 2). La compétence traductionnelle est une priorité pour elles. Huit étudiantes mettent en évidence l'intérêt de faire des projets linguistiques contre cinq étudiantes qui veulent travailler sur des projets variés. Huit étudiantes disent qu'elles n'ont pas de propositions des projets.

\section{Les types de projets dans une formation de traduction}

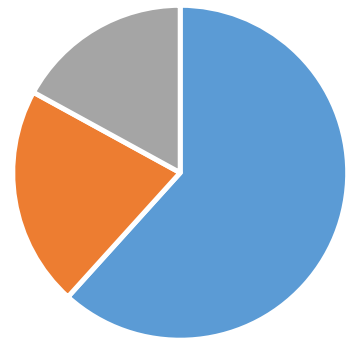

- projets de traduction - projets linguistiques - pas d'attente de projets

Figure 2 : les attentes des étudiantes vis-à-vis des types de projets à réaliser

Selon sept étudiantes, les projets de traduction doivent concerner la traduction des textes variés ; politiques, éducatifs, culturels et sociaux. Une étudiante exprime son désir de traduire une chanson, et quatre étudiantes souhaitent traduire des livres. Quatorze étudiantes ont dit que le nombre de projets de traduction doit être augmenté dans la formation. Pour vingt étudiantes, il s'agit de commencer à pratiquer la traduction de phrases simples dans les cours de langue aux premiers niveaux d'études, puis graduellement faire la traduction de paragraphes et de petits textes.

Parmi les projets à réaliser dans les cours de langue il y a l'écriture d'une histoire enfantine en français et sa traduction en arabe. D'ailleurs, deux étudiantes ont parlé de l'importance de traduire des textes ou des publicités de la faculté et de l'université. La traduction audiovisuelle n'est pas absente des attentes des étudiantes, elles estiment que c'est une activité utile. Un projet concernant les activités à réaliser en dehors de l'université est attendu par une étudiante qui souhaite acquérir des connaissances basiques sur la pratique de la traduction dans des instituts de traduction professionnelles.

Concernant les projets linguistiques, classés en deuxième lieu selon cette analyse, le développement des compétences linguistiques et communicatives a paru comme objectif des projets attendus. Neuf étudiantes ont dit qu'elles veulent travailler sur des projets d'écriture et d'expression orale pour améliorer et pratiquer la langue française. L'habilité de production est plus ciblée, selon leurs réponses, que celle de la réception.

XLinguae, Volume 12 Issue 1XL, January 2019, ISSN 1337-8384, eISSN 2453-711X 


\subsection{Analyse qualitative}

\subsubsection{Les descriptifs}

Les projets apparaissent dans neuf des vingt descriptifs. Ils sont présents dans les modules linguistiques : lecture et écriture, compétences à l'oral et grammaire appliquée. Les descriptifs des modules de traduction dans les cinq premiers niveaux n'incluent pas le terme projet. La place accordée aux projets varie selon les types de modules linguistiques ; ils sont des outils d'évaluation, parmi d'autres outils, dans les modules de lecture et d'expression écrite, et ceux des compétences à l'oral à tous les niveaux d'études (2-5). Ils sont notés pour 5\% de la note totale de chaque module sans présentation aux apprenants des critères d'évaluation des projets.

L'analyse des descriptifs de formation de traduction montre une absence d'intégration des projets dans les stratégies d'enseignement, et les cinq notes qui lui sont accordées sont faibles dans le cadre d'une formation linguistique visant les compétences professionnelles des futures traductrices en comparaison avec les quatre-vingt-quinze notes réparties sur d'autres éléments : les examens (intermédiaires 1, 2, et examen final), les devoirs, les quiz et la participation. En d'autres termes, la non-intégration du projet dans les processus d'enseignement influe sur les conduites des futures traductrices car, comme elles sont privées d'une expérience d'apprentissage où elles concrétisent leurs acquis à travers les projets, elles n'auront aucune expérience du travail en équipe et au sein des projets. D'un autre côté, les sujets des projets sont définis en termes de contenus proposés par le manuel du FLE ${ }^{\text {iv }}$, un manuel généraliste ne préparant pas les apprenants à la profession de traductrice car ses thématiques sont plutôt reliées aux situations de la vie quotidienne plus qu'à un domaine spécialisé, surtout pour les apprenants de niveaux A1 et A2.

\subsubsection{Les contenus des projets mis en œuvre}

Les projets mis en œuvre par les étudiantes, selon leurs réponses au questionnaire, comprennent des sujets sur la vie quotidienne sociale et professionnelle : écrire une annonce de vente de maison, décrire une personnalité célèbre ou influente, décrire une ville et des lieux célèbres, décrire un chemin, présenter sa candidature pour un emploi, faire un CV. D'autres contenus des projets se concentrent sur la traduction comme la traduction des textes du français en arabe, par exemple, des textes littéraires, un chapitre dans un livre, le résumé d'une histoire ou d'un film, le résumé d'un roman, l'élaboration d'un petit dictionnaire thématique et la traduction d'une vidéo courte.

\subsubsection{La cohérence des contenus des projets mis en œuvre avec les cadres de référence}

L'analyse des contenus ne pourrait être réalisée qu'en interaction avec les cadres de référence pour une meilleure compréhension de leur adéquation avec les objectifs de la formation et les niveaux des étudiantes.

\section{- Contenus des projets / compétences du référentiel de compétences de} l'EMT

Le référentiel de compétences de l'EMT établit les compétences nécessaires à la traduction professionnelle. Bien que la formation à la traduction soit une formation académique, il semble nécessaire d'intégrer des projets de traduction dès les niveaux 
linguistiques inférieurs des apprenants. Nous établissons un lien entre les contenus des projets mis en œuvre et les compétences du référentiel de compétences de l'EMT.

- Les compétences linguistiques : écrire une annonce de vente de maison, décrire une personnalité célèbre ou influente, décrire un chemin, faire un $\mathrm{CV}$, présenter sa candidature pour un emploi.

- Les compétences culturelles : décrire une ville et des lieux célèbres.

- Les compétences traductionnelles : traduire des textes du français en arabe (textes littéraires, un chapitre dans un livre), résumer une histoire, un film ou un roman, élaborer un petit dictionnaire thématique, traduire une vidéo courte.

- La présentation des services de traduction : élaborer une brochure touristique.

La recherche d'informations est une compétence transversale sans laquelle les étudiantes ne peuvent pas développer les autres compétences.

\section{- Contenus des projets/ guide de catégorisation des professions en Arabie Saoudite}

Le guide définit, en fonction du niveau de compétence du traducteur, trois types de profession à exercer après l'obtention de la licence en traduction : traducteur-assistant, traducteur, traducteur expérimenté. Il précise les domaines de spécialités : traduction orale et écrite de la langue arabe vers une ou plusieurs langues étrangères. Les textes à traduire sont des lettres officielles, des livres, des périodiques, des contrats, des rapports, etc. Les traducteurs doivent être capables d'exécuter toutes les tâches dans le domaine de traduction. Nous avons constaté que les connaissances disciplinaires en traduction et les connaissances linguistiques (grammaire et lexique) sont plus nombreuses que les compétences professionnelles. Les compétences sont essentiellement de deux types : les compétences rédactionnelles et les compétences en expression orale.

Les contenus des projets mis en œuvre répondent aux compétences du guide, comme les projets d'écriture ou de description d'une personne, d'un chemin. Mais aucun projet réalisé n'a un lien étroit avec les connaissances théoriques. Cela peut s'expliquer par le fait que la majorité des descriptifs traitent des modules linguistiques et très peu des modules théoriques de traduction. Une autre remarque est que le guide ne tient pas compte de la traduction orale ou de l'interprétation, ce qui peut être expliqué par un usage oral rare de la langue française en Arabie Saoudite, car l'anglais est devenu la langue la plus utilisée après la langue arabe dans ce pays.

\section{- Contenus des projets / niveaux linguistiques / activités de traduction dans le volume complémentaire du CECRL}

Les contenus des projets, qu'ils soient langagiers ou professionnels s'articulent autour des notions clés de compétences langagières visant la médiation des textes. Dans le cadre des projets mis en œuvre, des activités autres que celles de la traduction des textes ont été mises en œuvre, à savoir, les activités de traitement des textes dont l'objectif est de "résumer les points essentiels d'un texte source ; collecter des informations et des arguments de différentes sources ; identifier et expliquer au destinataire, le public visé, l'objectif et le point de vue de l'original" (CECRL, 2018: 117).

XLinguae, Volume 12 Issue 1XL, January 2019, ISSN 1337-8384, eISSN 2453-711X 
Les contenus des projets peuvent correspondre aux activités de médiation concernant la traduction à l'écrit d'un texte écrit en français, comme par exemple écrire une annonce de vente de maison, décrire une personnalité célèbre ou influente ou élaborer une brochure touristique. Les apprenants peuvent les traduire en arabe. Mais le choix de certains textes n'est pas toujours adapté au niveau des apprenants. Comme nous l'avons déjà évoqué, les étudiantes ont un niveau de $\mathrm{A} 1$ et $\mathrm{A} 2$, et les niveaux $\mathrm{B} 1$ ou B2 sont exigés pour la traduction des textes littéraires, d'un chapitre de livre et pour le résumé d'un film ou d'un roman de certains projets ce qui rend les projets plus difficiles à réaliser.

\section{Discussion}

Les catégories analysées nous conduisent avant tout à prendre en compte des éléments indissociables des composantes du référentiel tels que le niveau des étudiantes et les conditions d'opérationnalisation du référentiel.

Les niveaux des apprenants visés sont A1 et A2. Pour rendre les projets opérationnels et donner une efficacité à l'usage du référentiel, la place du projet doit être rétablie dans les descriptifs des modules linguistiques et de traduction. Le projet ne doit pas être seulement réalisé en dehors de la classe, mais il faut l'intégrer dans les cours de la formation. Sa fonction ne doit pas être seulement évaluative, le projet peut en effet être utilisé comme stratégie d'enseignement. La note accordée au projet peut être de vingt points et dans ce cas, le projet peut remplacer un des examens intermédiaires. Les modalités de sa mise en œuvre peuvent alterner le travail individuel et le travail collaboratif.

L'identification des composantes principales du référentiel des projets comprend les contenus, les compétences, les tâches et les critères d'évaluation.

\subsection{Les contenus}

Les contenus des projets doivent être définis autant que possible en fonction des attentes des étudiantes. Ils doivent inclure la traduction des mots, des expressions et des textes simples afin d'habituer les étudiantes avec ces types de documents qu'elles devront traduire. En revanche, pour les thématiques des textes variés destinés à la traduction, nous pensons qu'il est difficile de leur proposer de traduire des textes spécialisés en politique, en économique ou en littérature. Le niveau des étudiantes (A1 et A2) impose des contenus relatifs aux situations de la vie quotidienne, aux sujets familiers, aux nouvelles de personnes, aux courtes histoires, adresses, messages, aux instructions, aux expressions simples des panneaux, aux affiches, aux programmes, aux dépliants, aux mots, aux expressions et aux textes simples sur des thèmes quotidiens. La traduction des textes plus spécialisés doit être proposée en fonction de la progression des niveaux des étudiantes.

\subsection{Les compétences}

Les compétences visées par notre public concernent la capacité à traduire des mots, des expressions et des textes écrits en langue A (la langue française). La traduction est réalisée vers la langue cible $B$ (la langue arabe). Le volume complémentaire du CECRL définit les compétences en caractérisant la traduction et les documents sources. Il s'agit d'une traduction écrite approximative et compréhensible. Les textes de la langue source sont des textes écrits simples, relatifs aux sujets de la vie quotidienne (CECRL, 2018 : 118).

Pour la traduction orale en langue B (la langue arabe) de textes écrits en langue A (la langue française), les compétences définies par le CECRL (2018 : 118) évoquent deux 
types de documents sources, à savoir les textes et les informations courantes. La traduction orale doit être simple et sommaire. Les genres de textes à traduire sont explicités dans ce type de traduction. Il s'agit des textes informatifs, relatifs aux sujets familiers.

Ces compétences contribuent au développement de manière indirecte des compétences spécifiques en traduction (Hurtado Albir, 2008 : 35), comme par exemple, la compétence contrastive pour la connaissance des différences entre les deux langues. Elles développent la compétence extralinguistique visant à mobiliser des connaissances encyclopédiques, biculturelles et thématiques. Les autres compétences spécifiques sont la compétence instrumentale en rapport avec l'utilisation des outils utiles au traducteur et des sources de documentation, et la compétence textuelle en rapport avec la résolution des différents problèmes de traduction. Enfin, la compétence professionnelle qui permet d'agir avec efficacité dans le monde de travail. Les apprenants, à partir de la traduction de textes simples au début de leur apprentissage, acquièrent au fur et à mesure ces compétences au cours de leur formation linguistique.

Pourtant, les étudiantes ont besoin d'être formées aux compétences méthodologiques dans les cours de traduction. Notre raisonnement d'élaboration du référentiel implique une concentration sur la définition des catégories de compétences spécifiques qui forment la compétence de traduction comme point de départ pour l'élaboration du référentiel, car une formation à la traduction est conçue principalement dans le but d'aboutir à une insertion rapide dans le monde du travail.

\subsection{Les tâches}

Les tâches sont de deux types : les tâches langagières et les tâches de médiation préparatoires aux activités de l'exercice professionnel. Les tâches langagières concernent les quatre habiletés qu'un apprenant de langue étrangère doit maîtriser (lire, écrire, parler et écouter). Elles sont communicatives dans la mesure où l'apprenant s'engage dans un acte de communication avec un ou plusieurs interlocuteurs afin de répondre à un besoin dans une situation donnée (CECRL, 2001 : 46). Rappelons que la tâche doit avoir essentiellement une visée communicative, interactionnelle et pragmatique, pour donner un sens à l'apprentissage (Kandeel, 2010 : 86). Nous mettons l'accent dans le cadre du référentiel sur la tâche de médiation linguistique qui comprend (mais ne se résume pas à) la dimension inter-linguistique, notamment savoir comment traduire et interpréter de façon plus ou moins formelle, ou transformer un type de texte en un autre. Elle comprend aussi la dimension intralinguistique qui peut concerner la langue cible (par ex. résumer dans la L2 un texte en L2) ou la langue initiale, dont la langue maternelle. (North \& Piccardo, 2016 : 13).

Ce type de médiation implique une médiation culturelle pour assurer le passage d'une culture à une autre dans les langues de traduction.

\subsection{Les critères d'évaluation}

Les critères d'évaluation varient selon les types de tâches et la nature de compétences. Pour évaluer la production et l'expression orale et écrite, nous adoptons les grilles d'évaluation de ces activités et tâches d'apprentissage langagier préconisées par le CECRL. Pour évaluer les tâches de traduction, nous nous sommes inspirée des objectifs de la traduction de la langue étrangère vers la langue maternelle comme le 
propose Martinez Melis (2001 : 231-232) et de la grille d'auto-évaluation de la médiation de textes élaborés dans le volume complémentaire du CECRL (2018 : 178).

\section{La production d'un modèle de document du référentiel des projets}

Les résultats de l'enquête ont montré que les attentes des étudiantes vis-à-vis des types de projets à réaliser concernent ceux de la traduction. Il s'agit donc d'intégrer la traduction dans les projets proposés dans les premiers niveaux de formation. Le modèle présenté dans le tableau 7 (voir annexes) représente un document du référentiel des projets pour un cours de compréhension et expression écrites dispensé au cinquième niveau. C'est une écriture créative du manifeste d'une association à choisir par les étudiantes selon leurs intérêts (association des amies de la traduction, association des amis des personnes handicapées, etc.) ${ }^{\mathrm{V}}$ Selon le CECRL (2001 : 52), l'apprenant doit être capable d'écrire sur les aspects quotidiens de son environnement et de faire une description brève et élémentaire d'un événement, d'activités passées et d'expériences personnelles.

Les compétences à mettre en œuvre sont des compétences langagières, des compétences de médiation favorisant l'acquisition des compétences en traduction (voir annexes, tableaux 8 et 9). Les activités privilégiées de mise en œuvre de projet sont les tâches. Le projet est donc construit autour des tâches de production écrite et des tâches de traduction. Cette dernière est définie comme "une unité d'activités, représentative de la pratique de la traduction, qui s'adresse à l'apprentissage de la traduction et qui a un objectif concret, une structure et une séquence de travail" (Hurtado Albir, 1999, p. 56 cité par (Hurtado Albir, 2008 : 45). L'approche didactique que nous adoptons pour la conception des tâches est fondée sur les principes des théories constructivistes (cognitive et sociale) ; les apprentissages se réalisent dans l'action sociale afin de doter les étudiantes d'une compétence de traducteur. L'approche ne vise pas principalement à faire une traduction totalement correcte des mots, des phrases ou même des textes, mais de rendre les étudiantes capables de créer des textes acceptés au sein de la communauté concernée (Kiraly, 2000 : 13-14). Nous tenons compte non seulement des contenus, mais du but, du temps et des résultats escomptés de l'apprentissage car la traduction est avant tout une activité textuelle, communicative et cognitive. Notre objectif n'est pas de copier les contenus et les compétences indiqués dans les cadres de référence consultés dans cette étude, même s'ils ont fait leurs preuves ailleurs, mais de se fonder sur une acquisition de savoirs et d'habiletés requis pour savoir traduire.

En rédigeant un texte en français, et en le traduisant ensuite en arabe, l'apprenant a l'avantage "de mettre en présence d'une manière explicite les deux langues et de développer cette capacité indispensable pour un traducteur qui consiste à séparer les deux langues, à éviter les calques et les contaminations" (Martinez Melis, 2001 : 164). Les critères d'évaluation sont établis en fonction des types de tâches (voir annexes, tableau 10).

\section{Conclusion}

Nous avons essayé au cours de cette recherche d'identifier les composantes essentielles du référentiel des projets dans une formation de traduction destinée à un public du FLE. La recherche s'inscrit dans une démarche didactique permettant de créer un point de repère partagé dans l'activité formative des enseignantes. Elle incite à accorder une place plus importante aux projets que celle qui leur a été accordée dans la formation. Il s'agit de les relier aux problématiques professionnelles plutôt que de 
leur attribuer une fonction évaluative visant à vérifier des connaissances disciplinaires.

La présente étude ouvre des horizons à la réalisation d'autres recherches à réaliser pour l'élaboration du référentiel, et dans lesquelles nous comptons interroger les enseignants dans les formations de traduction au niveau national ainsi que les autres acteurs concernés par la qualité de la formation, comme les professionnels et les représentantes de l'enseignement supérieur. Il s'agit aussi d'examiner le degré de pertinence des composantes du référentiel dans le contexte de formation. Les résultats de l'ensemble des recherches pourraient constituer ainsi le noyau de l'élaboration du référentiel national des projets pour les départements francophones de traduction, dans le contexte universitaire saoudien.

This research was funded by the Deanship of Scientific Research at Princess Nourah bint Abdulrahman University through the Fast-track Research Funding Program.

\section{Bibliographic references}

BOUGHERRA, T. 1996. Pour une didactique des projets en FLE. In : Travaux de didactique du FLE, n. 36, pp. 98-108. ISSN : 0765-1635.

BRACKE, A. 2001. Activite langagiere et pédagogie de projet. In : Le Français dans le Monde : recherches et applications, numero special (juillet), pp. 175-187. ISSN : 0015-9395.

CADET, J.-P. 2014. Referentiels et activite : un rapport complexe a ameliorer. In : Formation emploi, revue française de sciences sociales, n. 128, pp. 101-128. ISSN : 2107-0946. Available online : http://formationemploi.revues.org/4328

CHAUVIGNE, C. 2010. Les referentiels en formation. In : Recherche et formation, n.64, pp.77-90. ISSN : 1968-3936. Available online :https://journals.openedition.org/rechercheformation/210

CHRISTIN, R. 1997. Le Projet pedagogique en francais. In: Le Francais dans le Monde, n. 288, pp. 24-28. ISBN: 978-2735208265

COMISSION EUROPEENNE. 2017. Referentiel de compétences de l'EMT. Available online :

https://ec.europa.eu/info/sites/info/files/about_the_european_commission/what_the_e uropean_commission_does/documents/emt_faq_fr.pdf

CONSEIL DE L'EUROPE 2001. Un Cadre Europeen Commun de Reference pour les Langues : Apprendre, Enseigner, Evaluer. Strasbourg : Division des Politiques

Linguistiques. ISBN 227805075-3.

CONSEIL DE L'EUROPE 2018. Cadre Européen Commun de Reference pour les Langues : Apprendre, Enseigner, Evaluer. Volume complementaire avec de nouveaux descripteurs. Strasbourg : programme des Politiques linguistiques. Available online : https://rm.coe.int/cecr-volume-complementaire-avec-de-nouveaux-

descripteurs/16807875d5

CROS, F. - RAISKY, C. 2010. Referentiel. In : Recherche et Formation, n. 64, pp. 105-116. ISSN: 1968-3936. Available online:

https://journals.openedition.org/rechercheformation/215

CUQ, J.-P. (dir.) 2003. Dictionnaire de didactique du français langue etrangere et

Seconde. Paris : CLE international. ISBN 978-2-09-033972-7.

GUERNIER, M.-C. 2012. Les contenus linguistiques dans les referentiels et les discours des formateurs. Definitions, conceptions et references. In : Lidil, vol. 45, pp. 73-92. ISSN :1960-6052. Available online : http://journals.openedition.org/lidil/3181 HAMEZ, M.-P. 2012. La pedagogie du projet : un interet partage en FLE, FLS et FLM. In : Le francais aujourd'hui, vol. 1, n. 176, pp. 77-90. ISBN 9782200927479. 
HURTADO ALBIR, A. 2008. Compétence en traduction et formation par competences. In: TTR: traduction, terminologie, redaction, vol. 21, n. 1, pp. 17-64. ISSN 0835-8443.

KANDEEL, R. 2010. Pour une nouvelle perspective d'apprentissage : l'approche par les taches dans un cours de français langue etrangere (FLE) a l'universite en Jordanie. In : la revue arabe des arts, vol 7, n. 2. pp. 85-98. ISSN 1818-9849. Available online : https://eis.hu.edu.jo/deanshipfiles/pub105531083.pdf\#page $=49$

KIRALY, D. 2000. A social constructivist approach to translator education. Manchester: St. Jerome Publishing. ISBN: 1900650339.

LECLERCQ, V. - VICHER, A. 2012. Etude sur les usages des referentiels dans la formation linguistique de base. In : Lidil, n.45. ISSN : 1960-6052. Available online :http://lidil.revues.org/3196

LENOIR, Y. 2010. La notion de referentialise dans la formation a l'enseignement. Apports et limites. In : Recherche et formation, n. 64, pp. 90-103. ISSN: 1968-3936. Available online : https://journals.openedition.org/rechercheformation/213

MARTINEZ MELIS, N. 2001. Evaluation et didactique de la traduction : le cas de la traduction dans la langue etrangere. Thèse de doctorat, Universitat Autonoma de Barcelona. ISBN : 8469978500.

MINISTERE DU SERVICE CIVIL. Guide de categorisation des metiers en Arabie Saoudite. Available online : https://www.mcs.gov.sa/ArchivingLibrary/Directory/DocLib2/new_bonood.pdf NORTH, B. - PICCARDO, E. 2016. Cadre Europeen Commun de Reference pour les Langues : Apprendre, Enseigner, Evaluer. Elaborer des descripteurs pour illustrer les aspects de la médiation pour le CECR. Strasbourg : programme des Politiques linguistiques. Available online: https://rm.coe.int/cadre-europeen-commun-dereference-pour-les-langues-apprendre-enseigne/168073ff32

PUREN, C. 2009. La nouvelle perspective actionnelle et ses implications sur la conception des manuels de langue. In : M.-L. Lions- Olivier et P. Liria (dir.), L'Approche actionnelle dans l'enseignement des langues, pp. 120-137. Barcelone : Diffusion Francais Langue Etrangere. ISBN 978-84-8443-545-7.

REY, B. 2010. Les referentiels. Entretien de Bernard Rey realise par Christian Chauvigne et Yves Lenoir. In : recherche et formation, n.64, pp. 117-120. ISSN : 1968-3936. Available online : https://journals.openedition.org/rechercheformation/219

Words : 8876

Characters : 59690 (33,2 standard pages)

Assoc. prof. Rana Kandeel, PHD.

Department of translation-French program, Faculty of languages

Princess Nourah bint Abdulrahman university

Riyadh, BOX:84428

Kingdom of Saudi Arabia (KSA).

RHKandeel@lpnu.edu.sa 


\section{Notes}

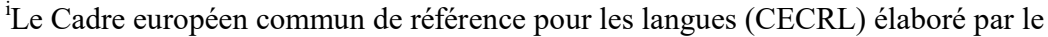
conseil de l'Europe en 2001 a toutes les caractéristiques d'un référentiel de formation. D'autres référentiels sont élaborés pour des finalités précises, à titre d'exemple :

- Chauver, A. (2008). Référentiel pour le Cadre Européen Commun. CLE

International : Paris.

- Beacco J.-C., de Ferrari M., Lhote G. et Tagliante C. (2005) : Niveau A1.1 pour le français. Référentiel et certification (Dilf) pour les premiers acquis en français, Paris,

Didier.

- Certification initiale de compétences en français, Centre de linguistique de

Besançon, université de Franche Comté, 2003.

${ }^{i i} L a$ licence est l'équivalent de Bachelor degree en anglais (bac+4).

${ }^{i i i}$ Guide de catégorisation des professions, disponible en arabe sur le site du ministère $\mathrm{du}$ service civil en Arabie Saoudite https://www.mcs.gov.sa/ArchivingLibrary/Directory/DocLib2/new_bonood.pdf

${ }^{\text {iv } H u g o t, ~ C . ~ e t ~ a l . ~(2012) . ~ A l t e r ~ e g o+, ~ m e ́ t h o d e ~ d e ~ f r a n c ̧ a i s, ~ H a c h e t t e ~ L i v r e ~: ~ P a r i s . ~}$

${ }^{\mathrm{V}}$ Le projet est à réaliser après avoir étudié un manifeste dans une leçon de la méthode Alter ego+ A2.

\section{Annexes}

Nom de projet

Création du manifeste d'une association en français et sa traduction en arabe

Type de traduction

Traduction générale (français-arabe)

Niveau visé

A2

Objectifs du projet

- s'entraîner à écrire un type textuel (le manifeste) en français.

- Traduire le manifeste en arabe.

Tableau 7 : définition du projet

Tâches de production écrite : rédiger en français le manifeste d'une association

Compétences langagières

-comprendre les objectifs de l'association et sa vision.

-connaître le vocabulaire thématique du manifeste (environnement, métier, social, etc.).

-utiliser les temps verbaux et les modes pour exprimer la nécessité (il est important/nécessaire/indispensable + sujet + verbe, il faut + verbe, vouloir que + subjonctif, etc.)

- parler des constats (constater que, être conscient que, etc.).

- informer des problèmes ou des difficultés.

- écrire des titres pour les sous-parties du manifeste.

- inciter à agir (impératif).

Tableau 8 : tâche et compétences langagières 
Tâche de traduction : traduire en arabe le manifeste d'une association

Compétences de médiation

- utiliser une langue simple et claire.

- faire une traduction approximative fondée sur la compréhension globale des expressions.

- traduire des textes très courts sur des thèmes quotidiens et familiers.

-respecter les exigences de la langue arabe (la morphosyntaxe, le vocabulaire, les connecteurs, l'orthographe et la ponctuation).

- Identifier et corriger des erreurs de traduction.

Compétences de traduction

-contrastives :

- comprendre le manifeste en français.

- $\quad$ produire le manifeste en arabe avec une langue simple.

- rechercher les différences entre les expressions et les phrases dans les deux langues (syntaxiques et sémantiques).

- Trouver l'équivalence de traduction.

-extralinguistiques:

- Identifier le contexte, la fonction et la forme du texte.

- Identifier les références culturelles, traduire les registres courants. -instrumentales:

- utiliser les dictionnaires en ligne pour rechercher le sens des mots et des expressions.

-textuelles:

- utiliser les sites fournisseurs des icônes ou des slogans.

- Identifier le plan du manifeste.

- Identifier les principaux traits stylistiques.

- utiliser des expressions en arabe pour constater, décrire des actions nécessaires et inciter à l'action.

- organiser les idées.

- professionnelles:

- comprendre la vision et les objectifs de l'association.

- identifier le public auquel s'adresse le manifeste.

- Créer un slogan pour l'évènement du manifeste.

- Imprimer le manifeste sous forme papier et électronique.

Tableau 9 : tâches et compétences de médiation et de traduction.

Critères d'évaluation sommative

Écriture du texte en français

Évaluer la capacité à :

- écrire un manifeste original.

- produire un texte simple et cohérent.

- utiliser des structures et des formes grammaticales simples relatives à la situation donnée.

- utiliser les registres de langue en adéquation avec le destinataire et le contexte.

- respecter le plan du manifeste. 
- décrire une situation.

- proposer des actions.

Traduction du texte en arabe

Évaluer la capacité à :

- transmettre les points principaux de textes courts et simples portant sur des sujets d'intérêt immédiat s'ils sont exprimés clairement en langue familière.

- reformuler de manière lisible et cohérente l'information.

- s'adapter au récepteur de la traduction.

- connaître le lexique relatif au thème de l'écriture.

- connaître la norme grammaticale.

- connaître les normes rédactionnelles.

- respecter les particularités du genre textuel.

Tableau 10 : critères d'évaluation des tâches 